\title{
tRNA-derived fragment tRF-03357 promotes cell proliferation, migration and invasion in high-grade serous ovarian cancer
}

\author{
Minmin Zhang ${ }^{1,2, *}$ \\ Feifei $\mathrm{Li}^{3, *}$ \\ Jing Wang \\ Wenzhu $\mathrm{He}^{\prime}$ \\ Yun Li' \\ Hongyan $\mathrm{Li}^{1}$ \\ Zhaolian Wei ${ }^{1,2,4}$ \\ Yunxia Cao ${ }^{1,2,4}$ \\ 'Department of Obstetrics and \\ Gynecology, The First Affiliated Hospital \\ of Anhui Medical University, Hefei, Anhui \\ 230022, People's Republic of China; \\ ${ }^{2}$ Anhui Province Key Laboratory of \\ Reproductive Health and Genetics, bio- \\ preservation and Artificial Organs, Anhui \\ Provincial Engineering Research Center, \\ Anhui Medical University, Hefei, People's \\ Republic of China; ${ }^{3}$ School of Basic \\ Medicine, Anhui Medical University, \\ Hefei, People's Republic of China; \\ ${ }^{4}$ Reproductive Medicine Center, The First \\ Affiliated Hospital of Anhui Medical \\ University, Hefei, Anhui 230022, People's \\ Republic of China \\ *These authors contributed equally to \\ this work
}

Correspondence: Yunxia Cao Department of Obstetrics and Gynecology, The First Affiliated Hospital of Anhui Medical University, No. 2I8, jixi road, Hefei, Anhui 230022, People's

Republic of China

Tel +86 I 3605605972

Email caoyunxia6@।26.com
This article was published in the following Dove Press journal: OncoTargets and Therapy

Background: High-grade serous ovarian cancer (HGSOC) is one of the most common ovarian epithelial malignancies. tRNA-derived fragments (tRFs) have been identified as novel potential biomarkers and targets for cancer therapy. Nevertheless, the influence of tRFs on HGSOC remains unknown. This study aimed to identify HGSOC-associated tRFs and to investigate the function and mechanism of key tRFs in SK-OV-3 ovarian cancer cells. Methods: The tRF profiles in HGSOC patients and controls were investigated using small RNA sequencing. Differentially expressed tRFs were verified by real-time PCR, and a key tRF was evaluated in function study.

Results: A total of 27 tRFs were differentially expressed between HGSOC patients and controls. Differentially expressed tRFs were mainly involved in the functions of protein phosphorylation, transcription and cell migration and the pathway of cancer, MAPK and Wnt signaling pathways. Real-time PCR verified that tRF-03357 and tRF-03358 were significantly increased in the HGSOC serum samples and SK-OV-3 cells compared to their expression levels in the controls. Importantly, tRF-03357 promoted SK-OV-3 cell proliferation, migration and invasion. Moreover, tRF-03357 was predicted targeted and significantly downregulated HMBOX1.

Conclusion: This study suggests that tRF-03357 might promote cell proliferation, migration and invasion partly by modulating HMBOX1 in HGSOC.

Keywords: high-grade serous ovarian cancer, tRNA-derived fragments, migration, invasion, cell growth

\section{Introduction}

Epithelial ovarian cancer is one of the deadliest malignancies among the gynecological malignant tumors. High-grade serous ovarian cancer (HGSOC) is the most frequent and deadly type of epithelial ovarian carcinoma and accounts for $75 \%$ of ovarian cancer cases. ${ }^{1}$ The onset of ovarian cancer is insidious, and the early stage lacks specific symptoms. More than $70 \%$ of ovarian cancer patients are diagnosed with advanced ovarian cancer. Under these conditions, the five-year survival rate of ovarian cancer patients drops below $30 \%{ }^{2}$ Currently, no specific peripheral blood screening method is available for ovarian cancer.

Transfer RNA-derived fragments (tRFs) are a novel class of noncoding RNA rooted in tRNAs ${ }^{3,4}$ that are 14-35 nucleotides (nt). ${ }^{5}$ Initially, tRFs were classified into $\mathrm{tRF}-5$, tRF-3, and tRF-1 in prostate cancer. ${ }^{6}$ An increasing number of studies have revealed that $\mathrm{tRF}$ play pivotal roles in cell proliferation, DNA damage response, tumor 
progression and neurodegeneration via regulation of gene expression. ${ }^{7,8}$ Since tRFs can bind to Argonaute proteins (similar to miRNAs) and Piwi proteins (similar to piRNAs), their disruption may play a key role in cancer by regulating gene expression at different levels. ${ }^{9}$ Recently, tRFs have been identified as novel potential biomarkers and cancer treatment targets. ${ }^{10}$ A tRF signature has been detected in ovarian cancer tissues. ${ }^{11}$ However, the tRF profiles in the peripheral blood of ovarian cancer patients remain unknown, and the role of tRFs in ovarian cancer remain unclear.

In the present research, we aimed to screen HGSOCrelated tRFs and explored the possible functions of key tRFs in ovarian cancer cells. Serum samples from HGSOC patients and healthy donors were analyzed using small RNA sequencing. Then, the effect of a differentially expressed tRF on ovarian cancer cells was assessed using the Cell Counting Kit-8 (CCK-8), Transwell and terminal deoxyribonucleotidyltransferse (TdT)-mediated biotin-16dUTP nick-end labeling(TUNEL) assays.

\section{Materials and methods}

\section{Ovarian cancer patients and data collection}

This study includes serum samples from 23 ovarian cancer patients and 18 healthy donors. Small RNA sequencing was performed with serum samples from three HGSOC patients and three controls. Information from the participants enrolled in the small RNA sequencing study is shown in Table S1. Real-time PCR was performed on serum samples from 20 ovarian cancer patients and 15 healthy controls; the characteristics of the patients are displayed in Table S2. Patients with hypertension, diabetes mellitus and infectious diseases were excluded in both groups. This study was approved by the ethics committee of the First Affiliated Hospital of Anhui Medical University. All women provided written informed consent.

\section{RNA isolation, library construction and small RNA sequencing}

Total RNA was isolated from the serum samples using the TRIzol reagent (Invitrogen, Carlsbad, CA, USA). The quality and quantity of the isolated RNA were measured using the Nanodrop 2000 (Thermo Fisher, MA, USA). The small RNA libraries were constructed and sequenced by Yingbio (Shanghai, China). Briefly, 3'- and 5'-adapters were combined with the small RNAs; then, complementary DNA (cDNA) was synthesized, and PCR was performed. RNA in the $135-170 \mathrm{nt}$ size range was excised by polyacrylamide gel electrophoresis and purified. After quantitative analysis and quality inspection, the six libraries were sequenced using the IlluminaHiSeq 2500 (Illumina, San Diego, CA, USA).

\section{Bioinformatics analysis}

Reads shorter than $15 \mathrm{nt}$ and with low quality were filtered out from the raw sequencing data. To identify tRFs, all clean reads were compared with the miRBase database (http://www.mir base.org/) to obtain identified known miRNAs. Sequences that could not be compared to the miRBase were mapped to the piwi-interacting RNA (piRNA) database to obtain piRNAs. Sequences that still were not mapped were mapped to the Genomic tRNA database (http://gtrnadb.ucsc.edu/) and tRFdb(http://genome.bioch.virginia.edu/trfdb/) to obtain the tRFs. Differentially expressed tRFs was identified based on a $\mid \log 2$-fold change $\mid>1$ and $\mathrm{FDR}<0.05$. miRanda (http:// www.microrna.org/microrna/home.do) and RNAhybrid (https://bibiserv.cebitec.uni-bielefeld.de/rnahybrid) was used to predict target genes of the differently expressed tRFs. The significantly target genes were obtained by intersecting miRanda (score $>150$; energy $<-20$ ) and RNAhybrid (energy $<-25$ ). The functions of these target genes were analyzed via gene ontology (GO). Pathways in which the target genes were enriched were identified using the Kyoto Encyclopedia of Genes and Genomes (KEGG) database.

\section{Real-time PCR}

RNA isolated from serum samples of 20 patients with ovarian cancer and 15 donors was reverse transcribed to cDNA using a reverse transcription kit(ThermoScientific, Madison, WI, USA). In brief, $1 \mu \mathrm{g}$ RNA was added with $1 \mu \mathrm{L} 3^{\prime}$ adaptor and nuclease-free water to a total volume of $7 \mu \mathrm{L}$, and incubated at $70{ }^{\circ} \mathrm{C}$ for $2 \mathrm{~min}$. The reactions were added with $10 \mu \mathrm{L}$ 3'ligation reaction buffer $(2 \mathrm{X})$ and $3 \mu \mathrm{L}$ $3^{\prime}$ ligation enzyme, and incubated at $25{ }^{\circ} \mathrm{C}$ for $60 \mathrm{~min}$, added with $1 \mu \mathrm{L} 3$ 'primer and nuclease-free water to $25.5 \mu \mathrm{L}$ volume, incubation for $5 \mathrm{~min}$ at $75^{\circ} \mathrm{C}, 15 \mathrm{~min}$ for $37^{\circ} \mathrm{C}$ and $25^{\circ} \mathrm{C}$, respectively. Productions were added with $1 \mu \mathrm{L}$ 5'adaptor, $1 \mu \mathrm{L} 5$ 'ligation reaction buffer (10X), $2.5 \mu \mathrm{L} 5^{\prime}$ 'ligation enzyme and incubatedat $25^{\circ} \mathrm{C}$ for $60 \mathrm{~min}$. Samples were then added with $8 \mu \mathrm{L}$ first strand synthesis reaction buffer, $1 \mu \mathrm{L}$ RNase inhibitor and $1 \mu \mathrm{L}$ reverse transcriptase, and incubated at $50{ }^{\circ} \mathrm{C}$ for $60 \mathrm{~min}$. The realtime PCR was performed using the ABI PRISM 7900 Sequence Detector system (Applied Biosystems, Foster City, CA, USA) based on the manufacturer's instructions. The primers are shown in Table 1. The relative expression levels of the tRFs were analyzed with the $2^{-\Delta \Delta C T}$ method. 
Table I Primer sequences

\begin{tabular}{|l|l|}
\hline tRF name & Sequence (5'-3') \\
\hline U6-F & CGATACAGAGAAGATTAGCATGGC \\
\hline U6-R & AACGCTTCACGAATTTGCGT \\
\hline tRF-07650-F & CAGATTGGTGGTTCAGTGGTAGA \\
\hline tRF-07650-R & AGTGCGTGTCGTGGAGTCG \\
\hline tRF-03357-F & GCAGCATTGGTGGTTCAGTG \\
\hline tRF-03357-R & AGTGCGTGTCGTGGAGTCG \\
\hline tRF-03358-F & GCAGCATTGGTGGTTCAGTG \\
\hline tRF-03358-R & AGTGCGTGTCGTGGAGTCG \\
\hline tRF-03357 mimics & CAUUGGUGGUUCAGUGGUAGAAUUCUCGC \\
\hline mimics N.C & UUGUACUACACAAAAGUACUG \\
\hline tRF-03357 inhibitor & GCGAGAAUUCUACCACUGAACCACCAAUG \\
\hline Inhibitor N.C & CAGUACUUUUGUGUAGUACAA \\
\hline
\end{tabular}

Abbreviation: N.C, negative control.

The results were normalized to the expression of the endogenous control gene U6 supported by previous studies. ${ }^{12,13}$

\section{Cell culture and transfection}

The ovarian cancer cell lines SK-OV-3 and HO8910 (iCell Bioscience Inc,Shanghai, China) and normal human ovarian surface epithelial cell line HOSEPIC (Typical Culture Preservation Commission CellBank, Chinese Academy of Sciences, Shanghai, China) were used in this study. The SKOV-3, HOSEPIC and HO8910 cells were cultured in McCoy's 5A medium (Catalog \#16600-082, Gibco, Carlsbad, CA, USA), DMEM F12 medium (Catalog \#10-092-CVR, Corning, Inc., Corning, NY, USA), and RPMI 1640 medium (Catalog \#10-040-CV, Corning, Inc.,), respectively, supplemented with $10 \%$ FBS and $1 \% \mathrm{P} / \mathrm{S}$ (Sangon Biotech, Shanghai, China). The cells were seeded into 6-well plates at a density of $3 \times 10^{5}$ cells per well and grown for $24 \mathrm{~h}$ in complete medium until the fusion rate reached $80-90 \%$. Then, the cells were rinsed with fresh medium and transfected with the tRF03357 mimics, mimics NC, tRF03357 inhibitor,or inhibitor $\mathrm{NC}$ using Lipofectamine $\mathrm{TM}_{2000}$ (Invitrogen, CA, USA) according to the manufacturer's protocol. The cells were cultured for $24 \mathrm{~h}$ and then used for the follow-up experiments.

\section{Cell proliferation assay}

Many methods are used to study cell proliferation. In this experiment, cell proliferation was examined with the
CCK- 8 method. Cells were harvested after $48 \mathrm{~h}$ of transfection and then seeded into 96-well plates (3599, Corning, NY, USA) at a density of $2 \times 10^{4}$ cells $/ \mathrm{mL}$. Each well had 6 replicates, and marginal wells were included with sterile water as a contrast. After culturing for 0, 24, 48, 72 or 96 h, CCK-8 (Beyotime, Shanghai, China) was added, and the OD (absorbance, $450 \mathrm{~nm}$ ) was measured.

\section{Transwell assays}

The cell migration assay was performed with $0.8 \mu \mathrm{m}$ 24-well Transwell chambers (FALCON), and the cell invasion assay was performed using BioCoat ${ }^{\mathrm{TM}}$ Matrigel ${ }^{\circledR}(0.8 \mu \mathrm{m}, 24$-well Transwell chambers; BioCoat). The cell culture medium was aspirated, and the cells were washed with an appropriate amount of $1 \mathrm{X}$ PBS containing $0.25 \%$ trypsin-EDTA. The cells were returned to the incubator for digestion for $3 \mathrm{~min}$. After the cells were resected and rounded, an equal amount of complete medium was added to terminate digestion. After centrifugation at $200 \times \mathrm{g}$ for $3 \mathrm{~min}$, the supernatant was removed, the cells were resuspended in serum-free McCoy's 5A medium, and the cell concentration was adjusted to $2 \times 10^{5}$ cells $/ \mathrm{mL}$. A total of $700 \mu \mathrm{L}$ of medium containing $10 \%$ serum was added to the lower chamber, and $500 \mu \mathrm{L}$ of the cell suspension was added to the upper chamber; then, the cells were incubated for $24 \mathrm{~h}$. The cells in the upper chamber were removed, and the cells in the lower section were stained with $0.1 \%$ crystal violet. Three random visual fields were observed under the microscope.

\section{TUNEL assay}

Cells were washed with $1 \times$ PBS once for $3 \mathrm{~min}$ and then fixed with $4 \%$ paraformaldehyde at room temperature for $30 \mathrm{~min}$. The cells were washed with 1X PBS 3 times for $3 \mathrm{~min} / \mathrm{time}$. The cells were incubated with $0.3 \%$ Triton $\times 100$ at room temperature for $20 \mathrm{~min}$. Then, the cells were washed with $1 \times$ PBS 3 times for $3 \mathrm{~min} /$ time. The TUNEL assay solution was prepared (TdT enzyme: fluorescent marker solution $=1: 9$ ). The cells were incubated with the TUNEL detection liquid at $37^{\circ} \mathrm{C}$ in the dark for $1 \mathrm{~h}$. The cells were washed with $1 \times$ PBS 3 times for $3 \mathrm{~min} / \mathrm{time}$. The PI dye solution was prepared (PI: $\mathrm{PBS}=1: 500$ ) and incubated with the cells for $5 \mathrm{~min}$ (at room temperature and away from light). The cells were observed and photographed under a fluorescence inverted microscope.

\section{Western blotting}

Total protein was isolated, and the protein concentration was measured using the BCA assay kit (Pierce Biotechnology, Inc., Rockford, IL, USA). Equivalent 
amounts of protein from each group were loaded onto the $10 \%$ SDS-PAGE gel. The proteins were transferred to membranes (Millipore, Bedford, MA, USA) and then incubated with an HMBOX1 primary antibody (1:2000; bs18047R, Bioss) or GAPDH antibody $(1: 1000 ; 60004-1-\mathrm{Lg}$, Proteintech) at $4{ }^{\circ} \mathrm{C}$ overnight. Next, the membranes were washed and incubated with goat anti-rabbit IgG-HRP for 1-2 h. An enhanced chemiluminescence reagent (Thermo Fisher Scientific, Waltham, MA, USA) was used to visualize the protein bands. A photograph was taken using the Chemi Doc MP system (Bio-Rad, Hercules, CA, USA), and the optical density was obtained via ImageJ software.

\section{Statistical analysis}

Each experiment was repeated 3 times. Comparisons were analyzed using Student's $t$-test (two-tailed) for two groups and one-way ANOVA followed by Tukey's post hoc test for three groups. The statistical significance level was set at $\alpha=0.05$ (two-sided). The mean $\pm \mathrm{SD}$ is displayed in the figures.

\section{Results}

\section{Overview of the small RNA sequencing}

To identify HGSOC-associated tRFs, serum samples from three HGSOC patients and three healthy subjects were subjected to small RNA sequencing. After quality control of the 6 sequencing libraries, an average of 10.36 million raw reads were obtained from each library, and approximately 8.9 million clean reads $(85.85 \%$; Table S3) with a length $>15$ nt were retained. Among the total clean reads, 14,823,768 (27.76\%) reads were mapped to miRNAs, 5,863,859 (10.98\%) were mapped to tRFs and $258,239(0.48 \%)$ were mapped to potential piRNAs and 7,048 (0.01\%) were mapped to known piRNAs (Tables S4). Six types of tRFs were found in the ovarian cancer and healthy control subjects ( 3 '-half, 5 '-half, itRF, tRF-1, tRF-3 and tRF-5), among which tRF-5 was the dominant tRF (occupying $62.18 \%$ and $62.48 \%$ in ovarian cancer patients and healthy controls, respectively), followed by $5^{\prime}$-half and itRF (Table S5).

\section{Differentially expressed tRFs between the HGSOC patients and healthy controls}

A total of 2,165 tRFs were expressed in the sera of the HGSOC patients and healthy controls. From these tRFs, 27 differentially expressed tRFs were identified between the HGSOC patients and healthy controls, including 22 and 5 tRFs that were up- and down-regulated in the HGSOC patients, respectively (Figure 1A and B). Through GO analysis, we found that the targeted genes of the differentially expressed tRFs were mainly involved in protein phosphorylation, cell migration, protein dephosphorylation and other processes (Figure 2A). After mapping all of the targeted genes to terms in the KEGG database, we found that the differentially expressed tRFs primarily participated in the pathway in cancer, MAPK signaling pathway, FoxO signaling pathway, and Wnt signaling pathway (Figure 2B).

\section{Validation of the differentially expressed tRFs}

To validate the differential expression based on the fold changes and abundances, 3 highly expressed candidate tRFs (tRF-07650, tRF-03357, and tRF-03358) were measured by real-time PCR in 20 HGSOC and 15 control samples. Our results showed that tRF-03357 and tRF03358 differed between the ovarian cancer patients and controls (Figure 3A). Therefore, tRF-03357 and tRF03358 were evaluated in ovarian cancer cells (SK-OV-3 and HO8901) and human ovarian epithelial cells (HOSEPIC) by real-time PCR. tRF-03357 $(P=0.047)$ and tRF-03358 $(P=0.027)$ expression in the SK-OV-3 and HO8910 cells was significantly increased compared with that of the HOSEPIC cells, and the fold change of tRF03357 was higher than that of tRF-03358 (Figure 3B). Therefore, tRF-03357 was examined in the subsequent experiments. After transfection with the tRF-03357 mimics, tRF-03357 expression in the HOSEPIC cells was significantly upregulated compared with that of the cells transfected with the mimics-NC (Figure 3C, $P=0.0001$ ). Conversely, tRF-03357 expression in SK-OV-3 cells transfected with the tRF-03357 inhibitor was significantly downregulated compared to that of the cells transfected with the inhibitor-NC (Figure $3 \mathrm{C}, P=0.002$ ).

\section{tRF-03357 promoted ovarian cancer cell proliferation, migration and invasion}

The effects of tRF-03357 on the proliferation of both HOSEPIC and SK-OV-3 cells were assessed. The CCK-8 assay results showed that the proliferation of HOSEPIC cells transfected with the tRF-03357 mimics gradually increased compared with that of the normal control (Figure 4A), whereas the proliferation of SK-OV-3 cells transfected with the tRF03357 inhibitor was significantly inhibited compared with that of the normal control (Figure 4B). Moreover, we examined the invasive ability of both SK-OV-3 and HOSEPIC cells using the Transwell invasion assay. Similar results were obtained 
A

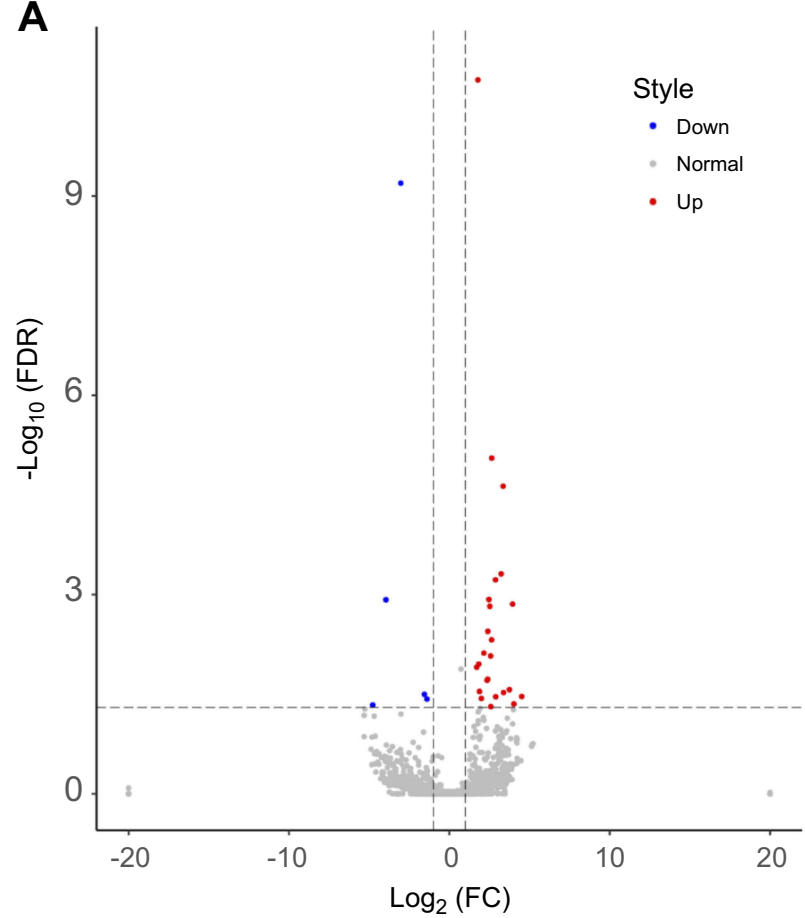

B

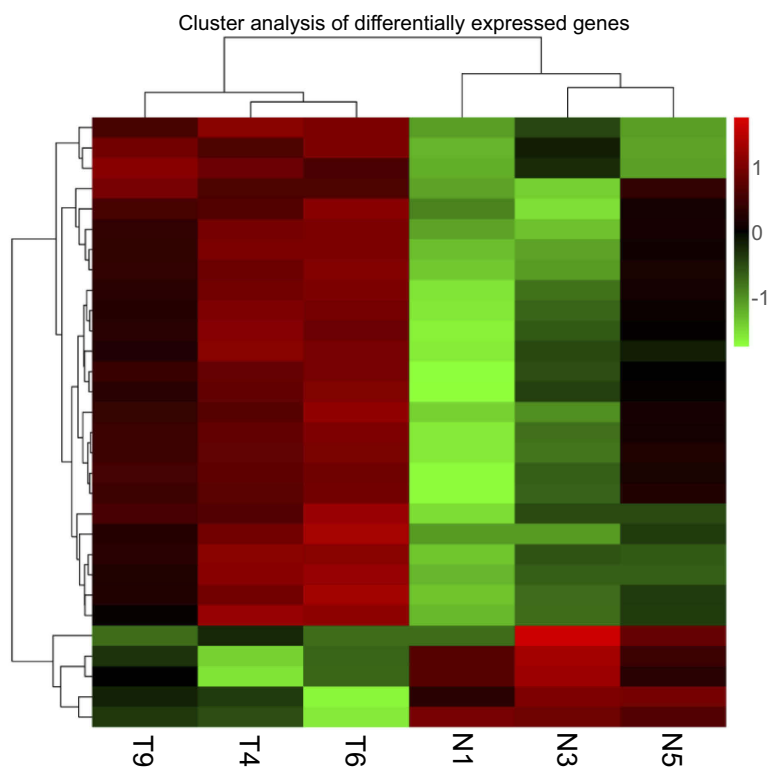

Figure I Differentially expressed tRFs between high-grade serous ovarian cancer (HGSOC) patients and three healthy subjects. (A) Volcano plot showing the differentially expressed tRFs between the ovarian cancer patients and healthy controls. The abscissa represents the fold change value, and the ordinate represents the FDR. Red dots indicate upregulated tRFs, and blue dots indicate down regulated tRFs. Gray dots represents not significant. Significantly different expression was identified based on a | log2fold change $|>|$ and FDR $<0.05$. (B) Cluster analysis showing the differentially expressed tRFs between the ovarian cancer patients and healthy controls. The T4, T6, and T9 groups belong to the tumor serum samples, and the NI, N3, and N5 groups belong to the normal serum samples. The enrichment factor increases from green to red.

that the number of migrating cells in the tRF-03357 mimics group was markedly increased compared to that of the control group (Figure 4C), whereas the number of migratory cells in the tRF-03357 inhibitor group was markedly weakened compared to that of the control group (Figure 4D). However, the TUNEL assay showed that the effect of tRF-03357 on apoptosis was not significant (Figures S1 and S2).

\section{tRF-03357 inhibited the expression of HMBOXI}

Five predicted target genes of tRF- 03357 were detected by real-time PCR. The results revealed that HMBOX1 was significantly increased by the tRF-03357 inhibitor, whereas PKN2, KLF3, PTPN13 and ESR2 were not significantly different (Figure 5A). As expected, the tRF03357 mimics significantly decreased the HMBOX1 mRNA level (Figure 5B). Similarly, the HMBOX1 protein expression level was increased by the tRF-03357 inhibitor and decreased by the tRF-03357 mimics (Figure 5C).

\section{Discussion}

HGSOC is one of the most common ovarian epithelial malignancies. It is usually diagnosed at an advanced stage and accounts for $75 \%$ of ovarian cancers. ${ }^{1}$ Accumulating evidence has shown that tRFs are critical regulators of cancer-related processes and may be novel diagnostic and therapeutic targets for tumor treatment. ${ }^{14,15}$ However, little is known about the roles of tRFs in ovarian carcinoma, especially HGSOC. This study is the first comprehensive and large-scale evaluation of tRFs in HGSOC. A total of 27 differentially expressed tRFs were identified between serum samples of HGSOC patients and healthy controls. The differentially expressed tRFs were mainly involved in the functions of protein phosphorylation and regulation of transcription and cell migration and the pathways of pathway in cancer, MAPK signaling pathway, FoxO signaling pathway, and Wnt signaling pathway. Moreover, real-time PCR verified that tRF-03357 and tRF-03358 were significantly increased in the HGSOC patients compared to their expression in the healthy controls. Further study showed that tRF03357 promoted SK-OV-3 cell proliferation, migration and invasion, as well as downregulated HMBOX1 expression.

The MAPK pathway, which is commonly known as the RAS-RAFMEK-ERK signal cascade, plays a vital role in multiple physiological processes, including cell proliferation, differentiation and apoptosis. ${ }^{16}$ The canonical MAPK 


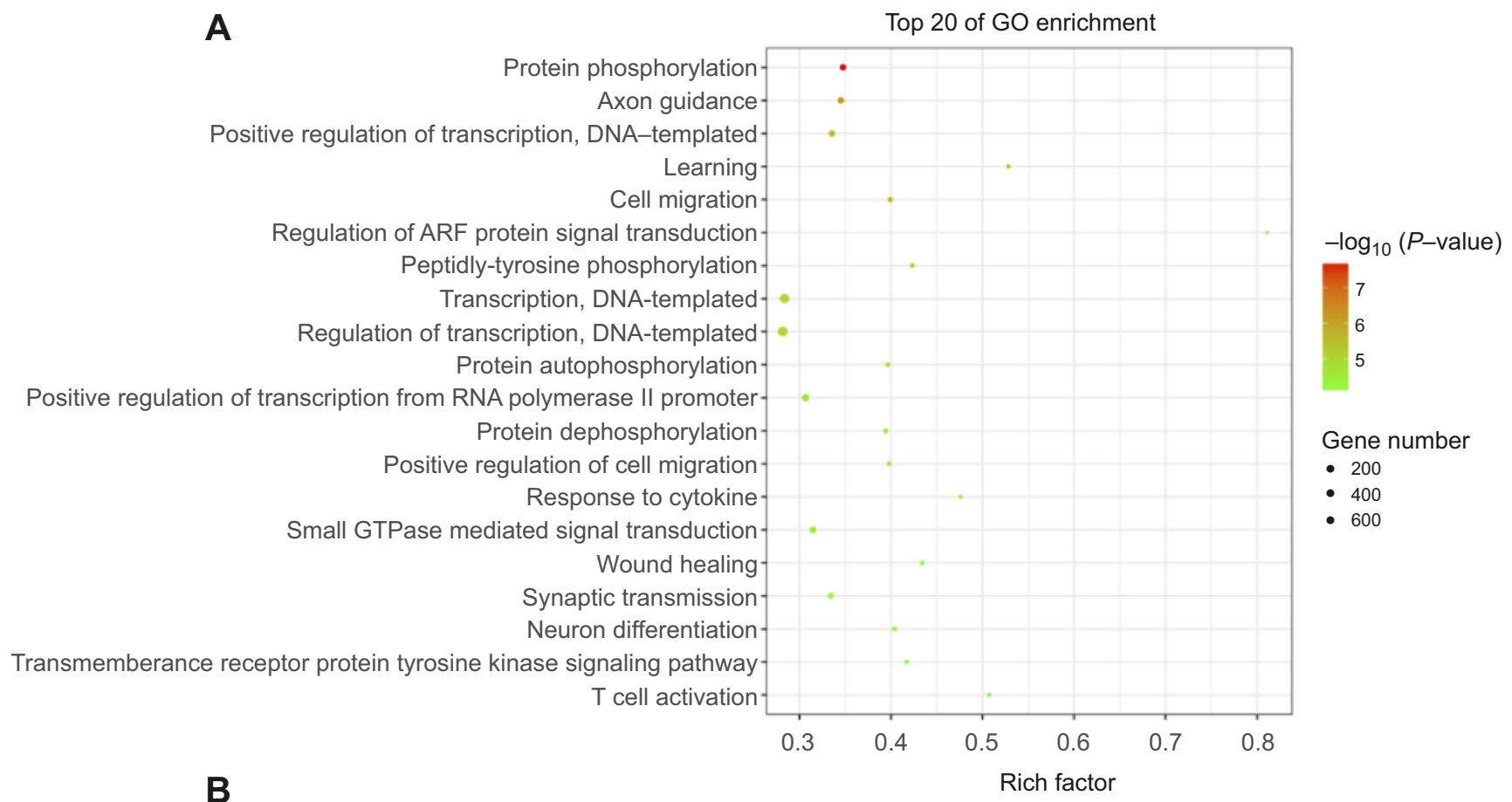

B

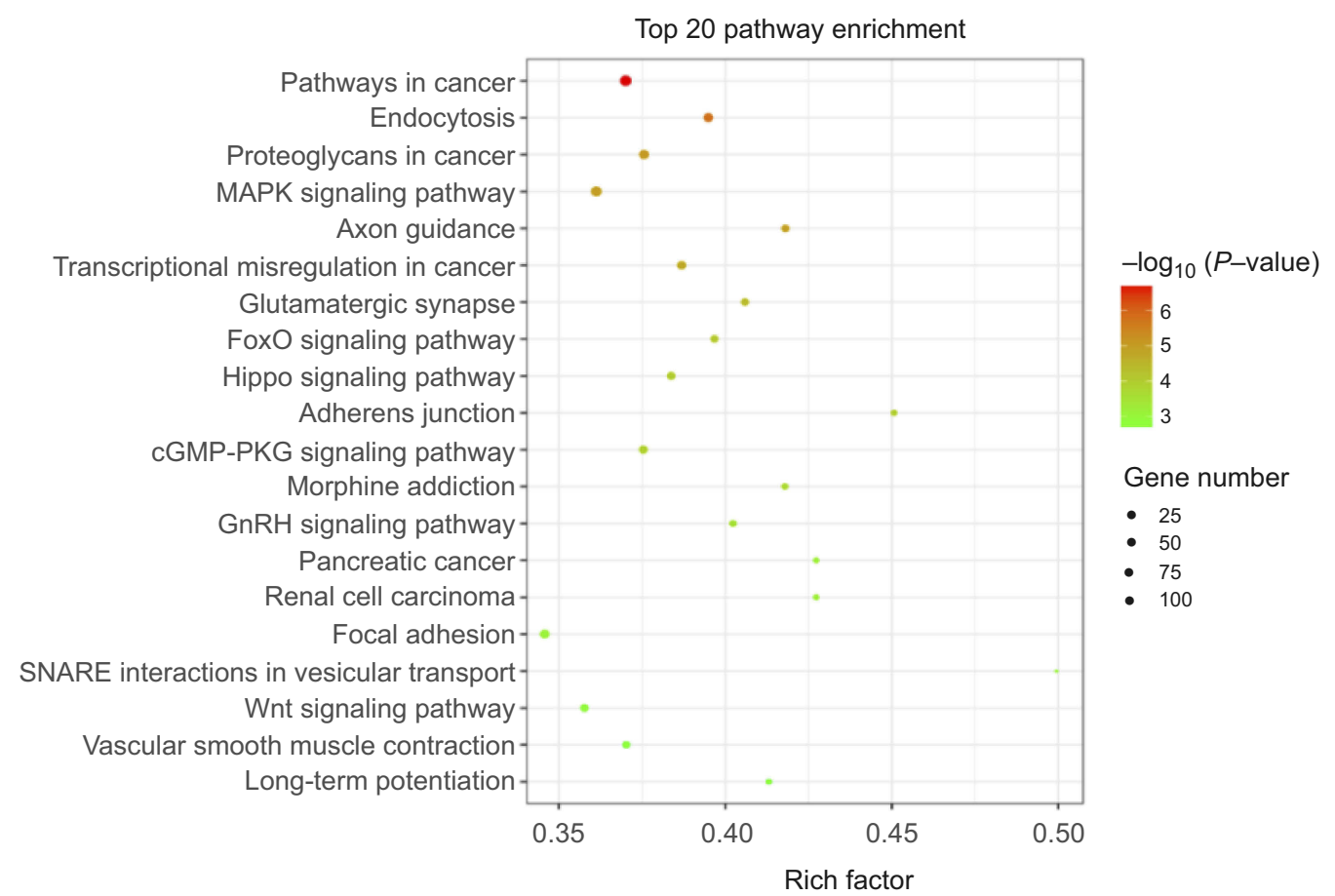

Figure 2 Function and pathway analysis of target genes of differently expressed tRFs between high-grade serous ovarian cancer (HGSOC) and three healthy subjects. (A) The top 20 enriched gene ontology (GO) terms of the differentially expressed tRF target genes. (B) The top 20 enriched KEGG pathways of the differentially expressed tRF target genes. Rich factor included the gene numbers and $P$-values.

pathway is activated by a combination of growth factors (such as EGF) and cell surface receptors, primarily tyrosine kinase receptors (RTKs, such as EGFR), which leads to dimerization and transphosphorylation of the RTKs. ${ }^{17}$ Aberrant activation of the MAPK pathway has been widely identified to be closely associated with a variety of cancers.
For instance, miR-98-5p inhibited tumor development by downregulating MAP4K4 and inhibiting the downstream MAPK/ERK signaling pathway. ${ }^{18}$ Downregulation of castor zinc finger 1 was related to a poor prognosis and promoted hepatocellular carcinoma progression via the MAPK/ERK pathway. ${ }^{19}$ PKP3 promoted ovarian cancer cell proliferation, 
A

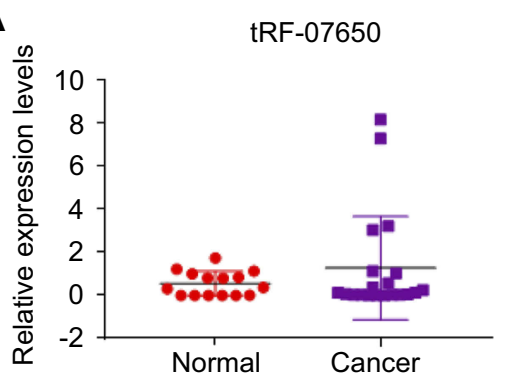

B

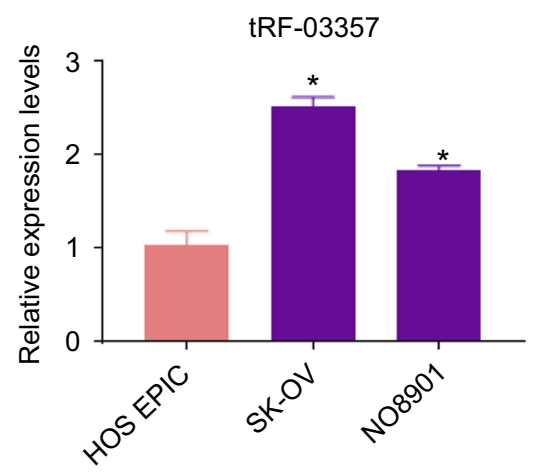

C

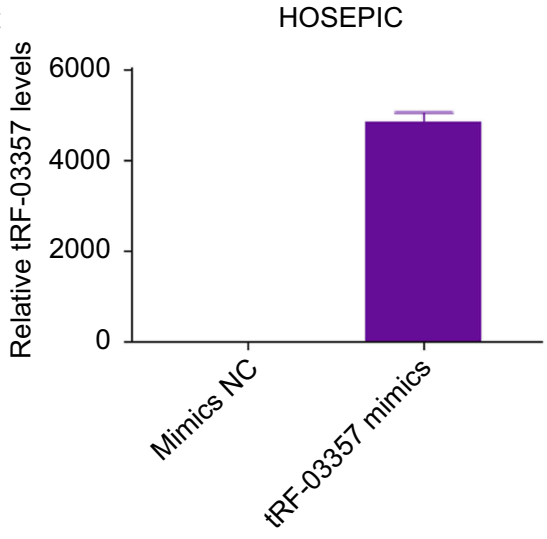

tRF-03357

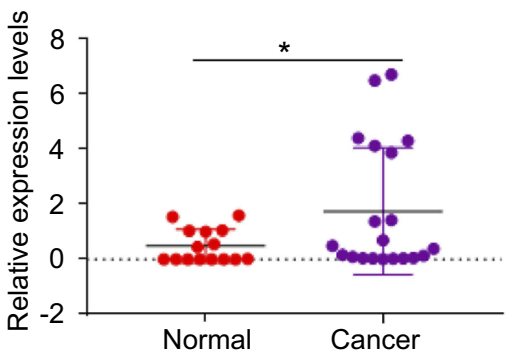

tRF-03358

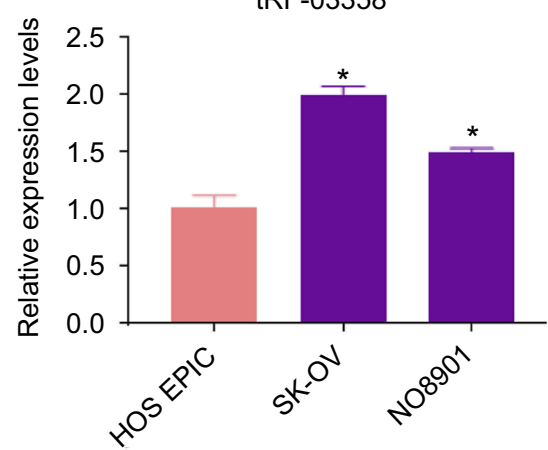

SK-OV-3

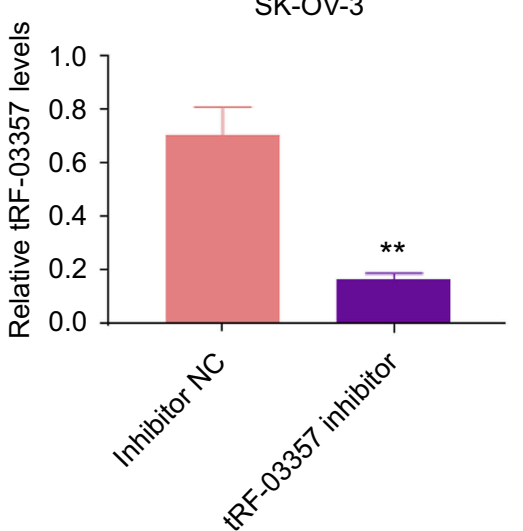

Figure 3 Verification of differentially expressed tRFs. (A) Candidate tRF expression (tRF-07650, tRF-03357, and tRF-03358) was measured by real-time PCR in serum samples from 20 high-grade serous ovarian cancer (HGSOC) patients and I 5 healthy controls; $t$-test. (B) tRF-03357 and tRF-03358 expression in ovarian cancer cells (NO890 I and SK-OV) and normal human ovarian epithelial cells (HOSEPIC) was measured by real-time PCR; one-way ANOVA followed by Turkey's post hoc test. (C) tRF-03357 expression in HOSEPIC cells transfected with the tRF- 03357 mimics or SK-OV-3 cells transfected with the tRF-03357 inhibitor was measured by real-time PCR; $t$-test. $* P<0.05$, $* * P<0.01$.

formation, and invasion through autophagy regulation via activating the MAPK-JNK-ERK1/2-mTOR pathway. ${ }^{20}$ Additionally, the Wnt signaling pathway plays a role in all three major gynecological cancers (ovarian, uterine and cervical). ${ }^{21}$ The Wnt ligands, including Wnt7A and Wnt7B, were reported to be increased in ovarian cancer and mediated tumor growth and progression via the WNT/beta-catenin pathway. ${ }^{22,23}$ This study showed that the targets of differentially expressed tRFs were mainly involved in the MAPK and Wnt signaling pathways, indicating that tRFs might modulate ovarian cancer progression via these pathways.
Recent studies have shown that tRFs are a new class of regulatory factors. Under cell stress, tRFs induced by ANG can induce the assembly of stress granules (SGs) and inhibit the synthesis of global proteins in a manner independent of eIF2 phosphorylation ${ }^{24,25}$ or by binding ribosomal proteins and fine tuning the protein synthesis rate. ${ }^{26}$ Moreover, tRNA methylation has been shown affect the stability and maturation of tRNAs. NSUN2 is a tRNA methyltransferase modifying tRNAs, and has been shown in association with human cancer prognosis such as ovarian cancer $^{27}$ and head and neck carcinoma. ${ }^{28}$ tRFs 

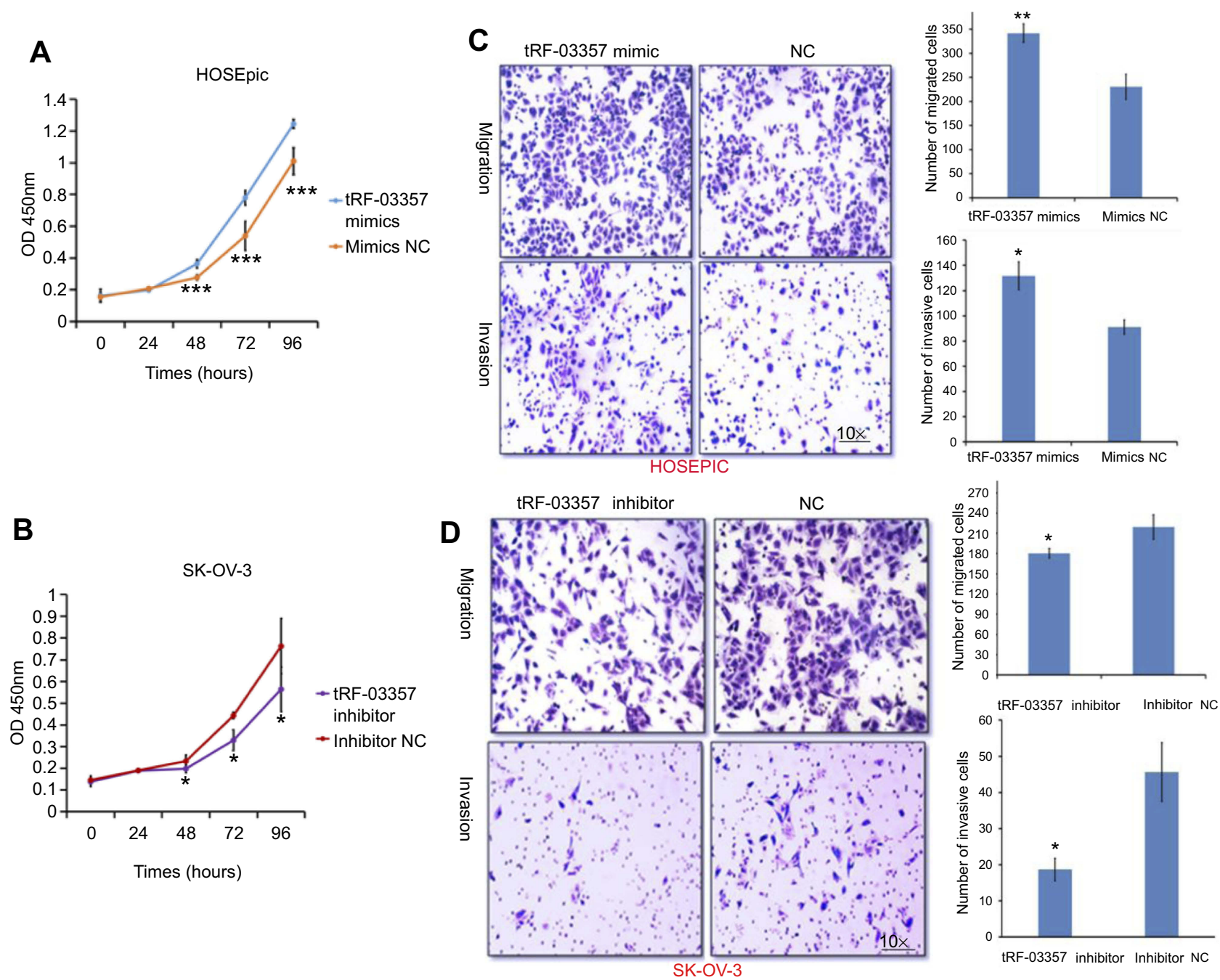

Figure 4 The effects of tRF-03357 on ovarian cancer cells. The effects of the tRF-03357 mimics (A) and inhibitor (B) on proliferation were detected using the CCK-8 assay. (C) The effects of the tRF-03357 mimics on HOSEPIC cell migration and invasion were evaluated using the Transwell assay. (D) The effects of the tRF-03357 inhibitor on SKOV-3 cell migration and invasion were evaluated using the Transwell assay.

Note: $* P<0.05$, $* * P<0.01$, $* * * P<0.001$

production has two approaches, a dicer-dependent and -independent manner. ${ }^{29}$ tRNA methylation has been shown to affect miRNA maturation, and maybe also for tRFs, given both share the similar maturation mechanisms. Moreover, tRFs have a mechanism that acts similarly to miRNAs, which can form a RISC complex with the Argonaute protein dependent on DICER generation and perform sequence-specific silencing of mRNA expression. For example, the generation of tRF-CU1276 is dependent on DICER1, which can bind four Argonaute proteins and can inhibit B lymphocyte proliferation and regulate DNA damage by silencing expression of its target gene RPA $1 .^{30}$ In this study, we found that tRF-03357 downregulated its predictive target gene HMBOX1, suggesting that tRF03357 might function similarly to a miRNA in ovarian cancer.
HMBOX1 is a transcription factor belonging to the hepatocyte nuclear factor family, which has been reported to be related to the development of several tumors. A previous study showed that HMBOX1 expression in HGSOC tissues and ovarian cancer cell lines was significantly lower than that in ovarian epithelial tissues or normal ovarian epithelial cell lines, and overexpression of HMBOX1 suppressed proliferation of the A2780 cell line. ${ }^{31}$ In human gliomas, the c-Fos/miR-18a feedback loop increased tumor growth via HMBOX1. ${ }^{32}$ In liver cancer, HMBOX1 inhibited tumor progression via elevating the autophagy level as well as suppressing stemness and immune escape ${ }^{33}$ However, in gastric cancer, HMBOX1 was upregulated and contributed to a poor prognosis by facilitating cell proliferation and migration, ${ }^{34}$ indicating distinct roles for HMBOX1 in different cancers. Our study showed that tRF03357 predictively targeted and downregulated HMBOX1 
A

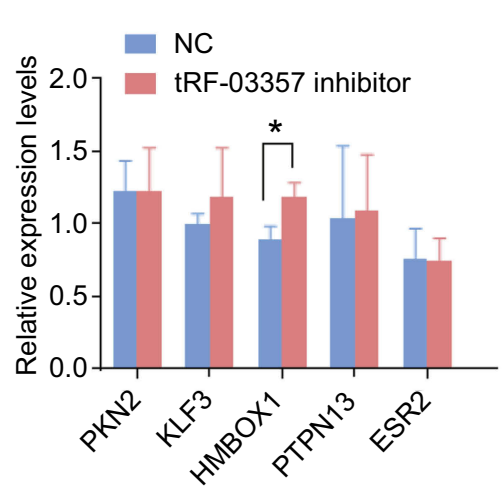

$\mathrm{B}_{\text {。으. }}$

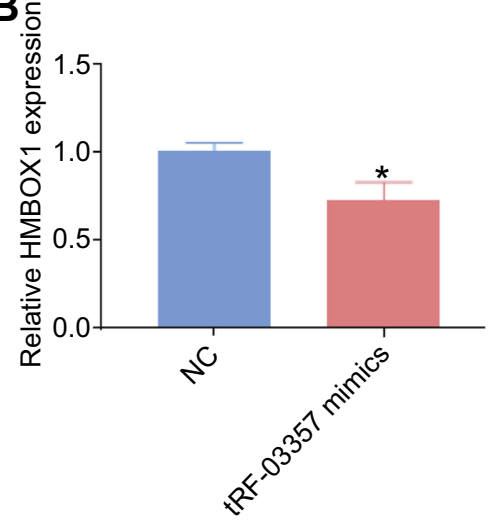

C

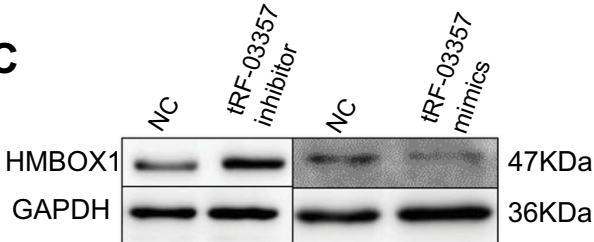

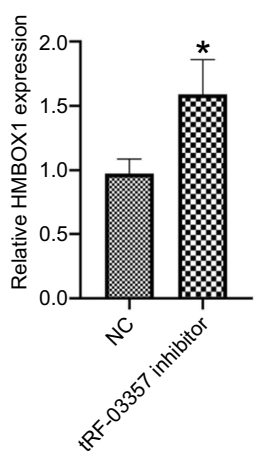

Figure 5 Target gene analysis for tRF-03357. (A) The expression of five predicted target genes of tRF-03357 were measured by real-time PCR in SK-OV-3 cells transfected with the tRF-03357 inhibitor and inhibitor NC. (B) HMBOXI expression was measured by real-time PCR in SK-OV-3 cells transfected with the tRF-03357 mimics and NC. (C) The HMBOXI protein level was measured by Western blotting in SK-OV-3 cells transfected with the tRF-03357 inhibitor or mimics.t-test, $* P<0.05$.

expression. These findings indicate that $\mathrm{tRF}-03357$ promotes the proliferation, migration and invasion of ovarian cancer might partly by downregulating HMBOX1.

\section{Conclusion}

In conclusion, tRF-03357 significantly promotes the proliferation, migration and invasion of ovarian cancer cells. This study might provide a potential diagnostic and therapeutic target for ovarian cancer.

\section{Acknowledgments}

This work was supported by the Central Government Special Funds to Guide the Development of Local Science and Technology (to Yunxia Cao).

\section{Disclosure}

The authors report no conflicts of interest in this work.

\section{References}

1. Ben-Baruch G, Sivan E, Moran O, Rizel S, Menczer J, Seidman DS. Primary peritoneal serous papillary carcinoma: a study of 25 cases and comparison with stage III-IV ovarian papillary serous carcinoma. Gynecol Oncol. 1996;60:393-396. doi:10.1006/gyno.1996.0060

2. Falconer H, Yin L, Gronberg H, Altman D. Ovarian cancer risk after salpingectomy: a nationwide population-based study. J Natl Cancer Inst. 2015;107. doi:10.1093/jnci/dju410

3. Kawaji H, Nakamura M, Takahashi $Y$, et al. Hidden layers of human small RNAs. BMC Genomics. 2008;9:157. doi:10.1186/ $1471-2164-9-157$
4. Cole C, Sobala A, Lu C, et al. Filtering of deep sequencing data reveals the existence of abundant Dicer-dependent small RNAs derived from tRNAs. RNA. 2009;15:2147-2160. doi:10.1261/ rna.1738409

5. Pekarsky Y, Balatti V, Palamarchuk A, et al. Dysregulation of a family of short noncoding RNAs, tsRNAs, in human cancer. Proc Natl Acad Sci U S S A. 2016;113:5071-5076. doi:10.1073/ pnas. 1604266113

6. Lee YS, Shibata Y, Malhotra A, Dutta A. A novel class of small RNAs: tRNA-derived RNA fragments (tRFs). Genes Dev. 2009;23:2639-2649. doi:10.1101/gad.1837609

7. Soares AR, Santos M. Discovery and function of transfer RNAderived fragments and their role in disease. Wiley Interdiscip Rev RNA. 2017;8. doi:10.1002/wrna.1423

8. Kumar P, Kuscu C, Dutta A. Biogenesis and function of transfer RNA-related fragments (tRFs). Trends Biochem Sci. 2016;41:679689. doi:10.1016/j.tibs.2016.05.004

9. Keam SP, Hutvagner G. tRNA-derived fragments (tRFs): emerging new roles for an ancient RNA in the regulation of gene expression. Life (Basel). 2015;5:1638-1651. doi:10.3390/life5041638

10. Balatti V, Pekarsky Y, Croce CM. Role of the tRNA-derived small RNAs in cancer: new potential biomarkers and target for therapy. $A d v$ Cancer Res. 2017;135:173-187. doi:10.1016/bs.acr.2017.06.007

11. Balatti V, Nigita G, Veneziano D, et al. tsRNA signatures in cancer. Proc Natl Acad Sci U S A. 2017;114:8071-8076. doi:10.1073/ pnas. 1706908114

12. Zheng G, Wang H, Zhang X, et al. Identification and validation of reference genes for qPCR detection of serum microRNAs in colorectal adenocarcinoma patients. PLoS One. 2013;8:e83025. doi:10.1371/journal.pone. 0083025

13. Zuberi M, Mir R, Das J, et al. Expression of serum miR-200a, miR$200 \mathrm{~b}$, and miR-200c as candidate biomarkers in epithelial ovarian cancer and their association with clinicopathological features. Clin Transl Oncol. 2015;17:779-787. doi:10.1007/s12094-015-1303-1

14. Cui Y, Huang Y, Wu X, et al. Hypoxia-induced tRNA-derived fragments, novel regulatory factor for doxorubicin resistance in triplenegative breast cancer. J Cell Physiol. 2019;234:8740-8751. doi: $10.1002 /$ jcp. 27533 
15. Sun C, Yang F, Zhang Y, et al. tRNA-derived fragments as novel predictive biomarkers for trastuzumab-resistant breast cancer. Cell Physiol Biochem. 2018;49:419-431. doi:10.1159/000492977

16. Liu F, Yang X, Geng M, Huang M. Targeting ERK, an Achilles' Heel of the MAPK pathway, in cancer therapy. Acta Pharm Sin B. 2018;8:552-562. doi:10.1016/j.apsb.2018.01.008

17. Huang L, Fu L. Mechanisms of resistance to EGFR tyrosine kinase inhibitors. Acta Pharm Sin B. 2015;5:390-401. doi:10.1016/j. apsb.2015.07.001

18. Fu Y, Liu X, Chen Q, et al. Downregulated miR-98-5p promotes PDAC proliferation and metastasis by reversely regulating MAP4K4. J Exp Clin Cancer Res. 2018;37:130. doi:10.1186/ s13046-018-0807-2

19. Wang JL, Yang MY, Xiao S, Sun B, Li YM, Yang LY. Downregulation of castor zinc finger 1 predicts poor prognosis and facilitates hepatocellular carcinoma progression via MAPK/ERK signaling. J Exp Clin Cancer Res. 2018;37:45. doi:10.1186/s13046-018-0720-8

20. Lim V, Zhu H, Diao S, Hu L, Hu J. PKP3 interactions with MAPKJNK-ERK1/2-mTOR pathway regulates autophagy and invasion in ovarian cancer. Biochem Biophys Res Commun. 2019;508:646-653. doi:10.1016/j.bbrc.2018.11.163

21. Ford CE, Henry C, Llamosas E, Djordjevic A, Hacker N. Wnt signalling in gynaecological cancers: a future target for personalised medicine? Gynecol Oncol. 2016;140:345-351. doi:10.1016/j. ygyno.2015.09.085

22. Yoshioka S, King ML, Ran S, et al. WNT7A regulates tumor growth and progression in ovarian cancer through the WNT/beta-catenin pathway. Mol Cancer Res. 2012;10:469-482. doi:10.1158/15417786.MCR-11-0177

23. King ML, Lindberg ME, Stodden GR, et al. WNT7A/beta-catenin signaling induces FGF1 and influences sensitivity to niclosamide in ovarian cancer. Oncogene. 2015;34:3452-3462. doi:10.1038/onc.2014.277

24. Yamasaki S, Ivanov P, Hu GF, Anderson P. Angiogenin cleaves tRNA and promotes stress-induced translational repression. J Cell Biol. 2009;185:35-42. doi:10.1083/jcb.200811106

25. Emara MM, Ivanov P, Hickman T, et al. Angiogenin-induced tRNAderived stress-induced RNAs promote stress-induced stress granule assembly. J Biol Chem. 2010;285:10959-10968. doi:10.1074/jbc. M109.077560
26. Mleczko AM, Celichowski P, Bakowska-Zywicka K. Transfer RNAderived fragments target and regulate ribosome-associated aminoacyl-transfer RNA synthetases. Biochim Biophys Acta Gene Regul Mech. 2018. doi:10.1016/j.bbagrm.2018.06.001

27. Yang JC, Risch E, Zhang M, Huang C, Huang H, Lu L. Association of tRNA methyltransferase NSUN2/IGF-II molecular signature with ovarian cancer survival. Future Oncol. 2017;13:1981-1990. doi:10.2217/fon2017-0084

28. Lu L, Zhu G, Zeng H, Xu Q, Holzmann K. High tRNA transferase NSUN2 gene expression is associated with poor prognosis in head and neck squamous carcinoma. Cancer Invest. 2018;36:246-253. doi:10.1080/07357907.2018.1466896

29. Kuscu C, Kumar P, Kiran M, Su Z, Malik A, Dutta A. tRNA fragments (tRFs) guide Ago to regulate gene expression post-transcriptionally in a Dicer-independent manner. RNA. 2018;24:10931105. doi:10.1261/rna.066126.118

30. Maute RL, Schneider C, Sumazin P, et al. tRNA-derived microRNA modulates proliferation and the DNA damage response and is downregulated in B cell lymphoma. Proc Natl Acad Sci U S A. 2013;110:1404-1409. doi:10.1073/pnas.1206761110

31. Yu YL, Diao NN, Li YZ, et al. Low expression level of HMBOX1 in high-grade serous ovarian cancer accelerates cell proliferation by inhibiting cell apoptosis. Biochem Biophys Res Commun. 2018;501:380-386. doi:10.1016/j.bbrc.2018.04.203

32. Zhou J, Wang M, Deng D. c-Fos/microRNA-18a feedback loop modulates the tumor growth via HMBOX1 in human gliomas. Biomed Pharmacother. 2018;107:1705-1711. doi:10.1016/j.biopha.2018.08.157

33. Zhao H, Jia H, Han Q, Zhang J. Homeobox containing 1 inhibits liver cancer progression by promoting autophagy as well as inhibiting stemness and immune escape. Oncol Rep. 2018;40:1657-1665. doi:10.3892/ or.2018.6551

34. Diao N, Li Y, Yang J, et al. High expression of HMBOX1 contributes to poor prognosis of gastric cancer by promoting cell proliferation and migration. Biomed Pharmacother. 2019;115:108867. doi:10.1016/j.biopha.2019.108867 


\section{Supplementary materials}

HOSEpic tsRNA03357 mimic

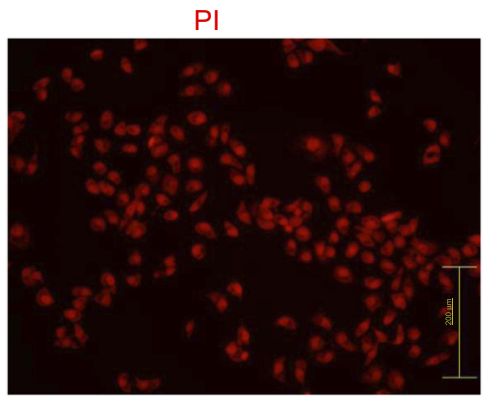

TUNEL
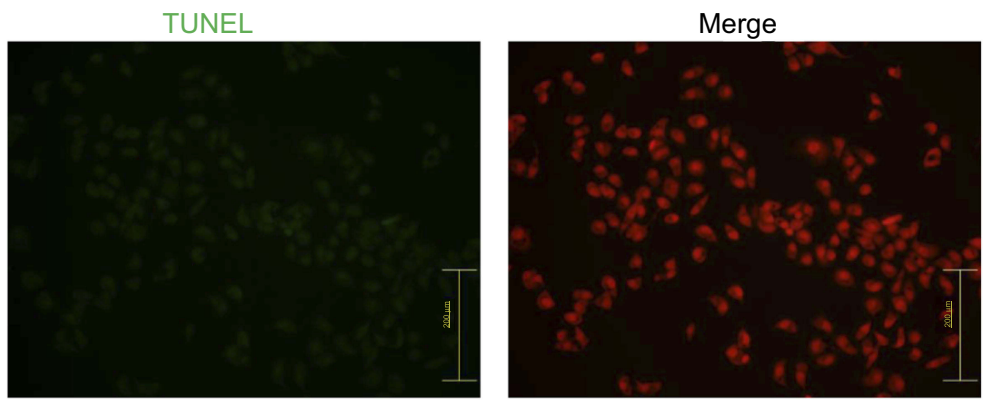

HOSEpic tsRNA03357 mimic NC
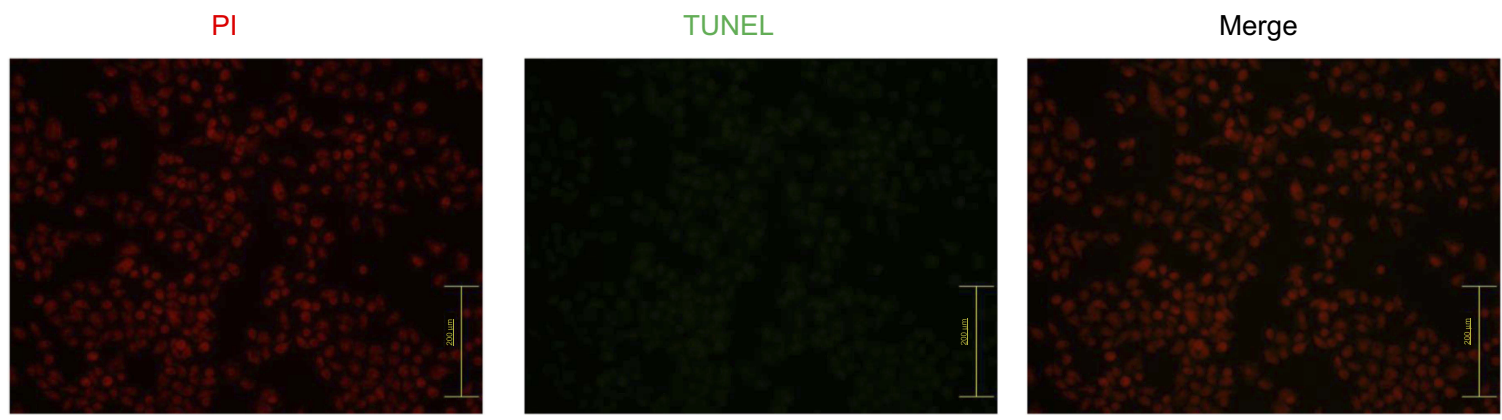

Magnification 200, scale bar $200 \mu \mathrm{m}$

Figure SI The effect of tRF-03357 mimic on apoptosis. The TUNEL assay showed that the effect of tRF-03357 on apoptosis in HOSEpiC transfected with tsRNA03357 mimic and mimic NC. Scale bar, $200 \mu \mathrm{m}$. 
SK-OV-3 tsRNA03357 inhibitor

PI

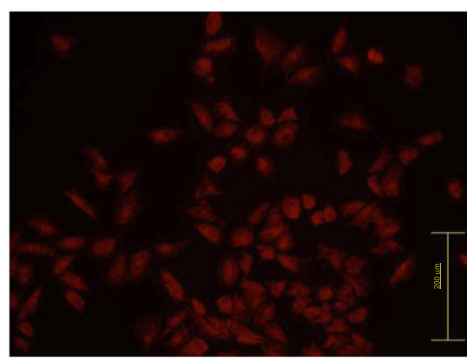

PI

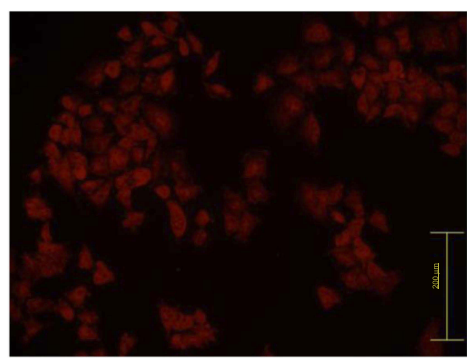

TUNEL

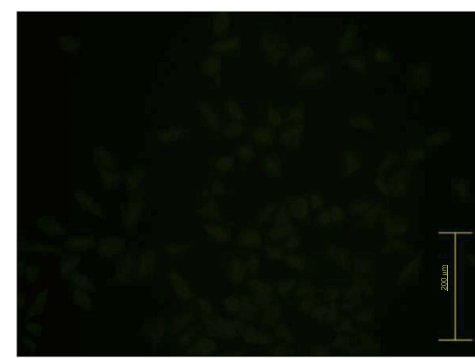

SK-OV-3 tsRNA03357 inhibitor NC

TUNEL

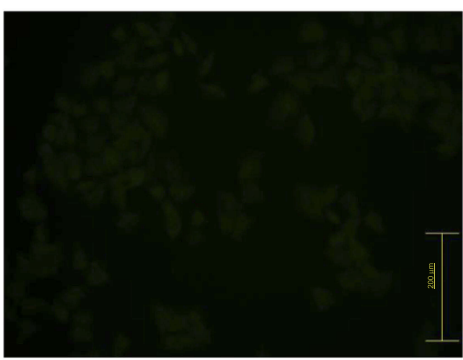

Migration 200, scale bar $200 \mu \mathrm{m}$
Merge

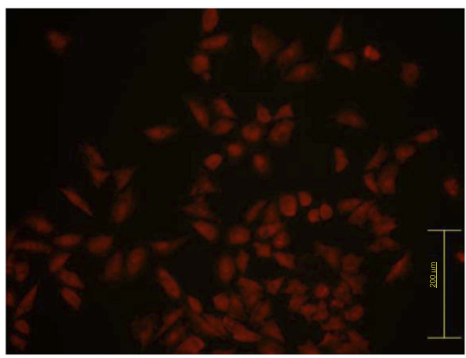

Merge

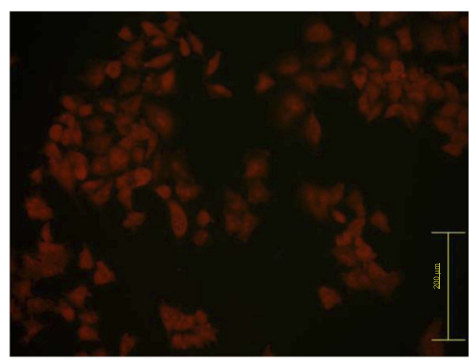

Figure S2 The effect of tRF-03357 inhibitor on apoptosis. The TUNEL assay showed that the effect of tRF-03357 on apoptosis in SK-OV-3 transfected with tsRNA03357 inhibitor and inhibitor negative control. Scale bar, $200 \mu \mathrm{m}$.

Table SI Information for participants enrolled in the small RNA sequencing analysis

\begin{tabular}{|l|l|l|}
\hline \multirow{2}{*}{ Characteristic (units) } & Patients $(\mathbf{n}=\mathbf{3})$ & Healthy controls $(\mathbf{n}=\mathbf{3})$ \\
\cline { 2 - 3 } & Average (range) & Average (range) \\
\hline Age (years) & $53(4 \mathrm{l}-68)$ & $58(53-64)$ \\
CAI25 (U/ml) & 2038.233 & - \\
HE4 (pmol/L) & 825.75 & - \\
Postoperative pathology & $3(100 \%)$ & - \\
\hline
\end{tabular}

Table S2 Information for the participants enrolled in the realtime PCR analysis

\begin{tabular}{|l|l|l|}
\hline $\begin{array}{l}\text { Characteristic } \\
\text { (units) }\end{array}$ & $\begin{array}{l}\text { Patients } \\
(\mathbf{n = 2 0})\end{array}$ & $\begin{array}{l}\text { Healthy controls } \\
(\mathbf{n}=15)\end{array}$ \\
\cline { 2 - 3 } & $\begin{array}{l}\text { Average } \\
\text { (range) }\end{array}$ & Average (range) \\
\hline Age (years) & $54(30-73)$ & $53(42-64)$ \\
\hline $\begin{array}{l}\text { Stages } \\
\text { I/II } \\
\text { III/IV }\end{array}$ & 3 & - \\
\hline CAI25 (U/ml) & 17 & - \\
\hline HE4 (pmol/L) & 715.724 & - \\
\hline
\end{tabular}


Table S3 Summary of cleaning data produced by small RNA sequencing

\begin{tabular}{|l|l|l|l|l|l|}
\hline Sample Name & Total reads & Clean reads (\%) & Total base & Clean base (\%) & GC (\%) \\
\hline T4 & $9,708,745$ & $7,988,48 I(82.28)$ & $485,421,762$ & $215,023,202(44.3)$ & 50 \\
T6 & $12,607,230$ & $11,178,587(88.67)$ & $630,350,747$ & $319,724,120(50.72)$ & 52 \\
T9 & $9,097,353$ & $8,747,774(96.16)$ & $454,851,084$ & $244,606,278(53.78)$ & $5 I$ \\
NI & $9,548,108$ & $7,335,84 I(76.83)$ & $477,383,449$ & $195,144,311(40.88)$ & 52 \\
N3 & $12,329,29 I$ & $9,437,165(76.54)$ & $616,459,997$ & $249,301,925(40.44)$ & $5 I$ \\
N5 & $8,914,165$ & $8,713,259(97.75)$ & $445,693,040$ & $230,707,426(51.76)$ & 49 \\
Average & $10,367,482$ & $8,900,185(85.85)$ & $518,360,013$ & $242,417,877(46.98)$ & $5 I$ \\
\hline
\end{tabular}

Table S4 Clean reads mapped to different small RNAs

\begin{tabular}{|l|l|l|l|l|l|}
\hline Sample & $\begin{array}{l}\text { Reads mapped to } \\
\text { miRNAs }\end{array}$ & $\begin{array}{l}\text { Reads mapped to } \\
\text { tRF }\end{array}$ & $\begin{array}{l}\text { Reads mapped to } \\
\text { potential }\end{array}$ & $\begin{array}{l}\text { Reads mapped to known } \\
\text { piRNA }\end{array}$ & $\begin{array}{l}\text { Total clean } \\
\text { reads }\end{array}$ \\
\hline T4 & $1,913,387$ & $1,601,320$ & 29,434 & 903 & $7,988,481$ \\
T6 & $1,736,544$ & $2,419,957$ & 101,606 & 3475 & $11,178,587$ \\
T9 & $2,525,167$ & 656,814 & 29,164 & 611 & $8,747,774$ \\
N1 & $1,453,347$ & 97,550 & 39,152 & 521 & $7,335,841$ \\
N3 & $3,010,426$ & 334,475 & 49,602 & 872 & $9,437,165$ \\
N5 & $4,184,897$ & 753,743 & 9281 & 666 & $8,713,259$ \\
Total & $14,823,768(27.76 \%)$ & $5,863,859(10.98 \%)$ & $258,239(0.48 \%)$ & $7048(0.01 \%)$ & $53,401,107$ \\
\hline
\end{tabular}

Table S5 Total counts of different tRFs in ovarian cancer $(T)$ and healthy controls $(\mathrm{N})$

\begin{tabular}{|l|l|l|}
\hline type & $\mathbf{T}(\%)$ & $\mathbf{N}(\%)$ \\
\hline i- tRF & $93,774(2.00 \%)$ & $38,787(3.27 \%)$ \\
tRF-3 & $8337(0.18 \%)$ & $1 \mathrm{I}, 642(0.98 \%)$ \\
tRF-5 & $2,909,058(62.18 \%)$ & $740,864(62.48 \%)$ \\
3'-half & $2321(0.05 \%)$ & $2715(0.23 \%)$ \\
$5^{\prime}$-half & $1,661,349(35.51 \%)$ & $387,030(32.64 \%)$ \\
tRF-I & $3252(0.07 \%)$ & $4730(0.40)$ \\
\hline
\end{tabular}

\section{Publish your work in this journal}

OncoTargets and Therapy is an international, peer-reviewed, open access journal focusing on the pathological basis of all cancers, potential targets for therapy and treatment protocols employed to improve the management of cancer patients. The journal also focuses on the impact of management programs and new therapeutic agents and protocols on patient perspectives such as quality of life, adherence and satisfaction. The manuscript management system is completely online and includes a very quick and fair peer-review system, which is all easy to use. Visit http://www.dovepress.com/ testimonials.php to read real quotes from published authors. 